Dr. Russell maintains the view that the "heart thas no reserve power to withstand a pressure of more than about 20-30 mm. above the mormal. Physiologists know, however, that the blood pressure can easily be doubled in an animal by the injection of suprarenal extract or excitation of the vaso-motor system. The hypertrophied heart must have far greater power than the normal heart. Dr. Williamson has attempted to substantiate this opinion (or deduction) of Dr. Russell by observations on the bloodpressure after forced exercise. I would point out that his observations are few in number and give no certainty that the normal resting pressure was obtained. Dr. Martin W. Flack and $I$ have published in the Journal of Physiology a series of observations on the blood-pressure of runners, boxers, and students after very severe stair-climbing exercise. The pressures were taken with my new sphygmometer, which enables one to take the pressure in the radial very quickly and immediately after the exercise. We found pressures rising in some cases to 220 millimetres and double the normal resting pressure in several cases. Inhalation of oxygen, too, made the artery fuller and the pressure higher. In students in hot baths we have found the pressure fall to $65 \mathrm{~mm}$. $\mathrm{Hg}$, so that we have a range from 65 to $220 \mathrm{~mm}$. $\mathrm{Hg}$ in normal men under these extreme conditions. In my own case recently during a headache I had a pressure of $165 \mathrm{~mm}$. when resting lying down, falling to $100 \mathrm{~mm}$. when the headache cleared off and with no sign of a contracted artery. On asking my colleagues the clinicians whether they have felt such rigid contracted arteries as Dr. Russell describes I have not obtained any affirmation, nor have I yet been able to find such a case to make measurements upon by my methods. I wish Dr. Russell would set such a case before me. Dr. Russell does not quite grasp, I think, the physiology of our "gravity method" when he compares the "leg up" readings of our three subjects. The men were of unequal stature, their legs were hoisted up to unequal extents and the vital reaction of the vaso-motor system to the change of posture was not necessarily the same in each. 'The whole point of this experiment is that the measurements in arm and leg came out fairly well in comparison with the column of blood separating the two places of measurement. This is a method which can very easily be applied to pathological cases and will yield, I think, results of great interest, for it will show us how far the compensation for gravity is disturbed in various morbid states. Dr. Flack and I find the cerebral pressure in the normal student is kept fairly constant in all three postures-horizontal, standing, and suspended head down. On rising from a very hot bath the compensatory mechanism does not act, the pulse accelerates, and the pressure falls greatly and the student becomes faint.

Dr. Williamson says that he has found marked inequality of pressures in the leg and arm in the horizontal posture in various pathological cases. I would ask whether these pressures were measured simultaneously, with one armlet on the arm and the other on the leg connected with one manometer, and the observer feeling radial and posterior tibial at the same time. It is essential that this should be done because (1) the systolic pressure of some pathological cases often varies with successive beats; (2) obliterating the leg may affect the pressure more than obliterating the arm; and (3) the pain produced by the armlet in cases of high pressure often is sufficient to make a difference to the pressure. found recently that pain produced by keeping the armlet on too long raised the pressures $20 \mathrm{~mm}$. in a case where at $210 \mathrm{~mm}$. both radial and posterior tibial were obliterated together. I make no dogmatic statement, for I think it possible that rigid arteries may be found by the gravity method which disturb the accuracy of the readings, although up to the present $I$ have found none. I am no partisan and defend no position but the establishment of the truth in this matter. We must bear in mind that Hürthle and others have found the systolic wave to be higher in the femoral than in the carotid of dogs. As to our "venous method," which I claim to be the most convincing method of proof, Dr. Russell seeks to overthrow this by measurements he has made with Dr. George Oliver's hæmomanometer. Now the first part of our inquiry was directed towards testing the accuracy of small unenclosed bags by this very vein method, and we quickly reached the conclusion that the application of such a bag to a vein was a proceeding which could only lead to error. The pressure of a small unenclosed bag cannot be applied so as reach the vessel in its full force. The armlet method is the only method by which the veins can be compressed and, contrary to what Dr. Russell says, it was quite easy for us to raise the pressure much higher than the pressure in the upper armlet and then let go for a time so that the veins could fill, and raise the pressure again, and so on, and we never found the pressure in the veins rise above that in the upper armlet. The ordinary laws of physics would be seriously upset if it did do so. Dr. Russell by his finding twice this pressure in the veins has only proved that Dr. Oliver's hæmomanometer cannot be used for the purpose to which he has applied it. We have just done two more cases, one of $185 \mathrm{~mm}$., and find the pressures correct by the vein method.

Loughton, March 14th, 1909.

I am, Sir, yours faithfully,

\section{THE MEDICAL DEFENCE UNION AND INSURANCE AGAINST LEGAL RISKS.}

\section{To the Editor of THE LANCET.}

SIR,-In consequence of the demand which has lately sprung up for insurance of medical practitioners against the risk of pecuniary loss when adverse verdicts, involving payment of plaintiff's costs and "damages," are returned against them in civil actions in their professional work, the council of the Medical Defence Union has concluded arrangements with a company of the very highest repute, "The Yorkshire Insurance Co.," for such indemnity. All members of the Medical Defence Union can now by payment of a yearly premium of the small sum of $7 s$. 6d. effect insurance up to the limit of $£ 2000$, and for a payment of $9 s$. up to the limit of $£ 2500$, in any cases defended by the Union in this respect. For fuller particulars and general conditions members are referred to the Secretary of the Yorkshire Insurance Co. (founded 1824), Bank Buildings, Princes-street, E.C., to whom all applications by members of the Union for policies should be made.

The Medical Defence Union will, as heretofore, guarantee the legal expenses connected with the defence of their members in any case taken up by the Council, and conduct such defence through their solicitor and counsel; the Yorkshire Insurance Co. will, in addition, guarantee the costs ordered to be paid and any damages up to $£ 2000$ or $£ 2500$ respectively in any individual action in which a verdict adverse to the member defendant is obtained. Thus a complete guarantee against pecuniary loss up to these limits to an individual member of the Medical Defence Union is secured in this respect; and further, by effecting the insurance the members of the medical profession will, I hope, no longer be harassed by appeals to their charity for defraying the expenses incurred when actions are recorded against the individual practitioner concerned; and this will undoubtedly be a great gain individually and collectively.

I am, Sir, yours faithfully,

A. G. BATEMAN,

General Secretary, Medical Defence Union.

4, Trafalgar-square, W.C., March 19th, 1909.

\section{TO MEDICAL FREEMASONS. To the Editor of THE LANCET.}

SIR,-May I once again, through the medium of your widely read columns, appeal to medical men, and medical Freemasons in particular, for votes for the Masonic charities. At the forthcoming elections in April there are two medical candidates for the Royal Masonic Institution for Boys. Votes for either institution are equally valuable and will be gratefully received and acknowledged by me.

I am, Sir, yours faithfully,

WILLIAM WILSON,

Secretary, St. Luke's Medical Lodge of Instruction. 184, Goldhawk-road, W., March 24th, 1909.

The Belgrave Hospitat.-The Belgrave Hospital for Children, Clapham-road, is making a special appeal for funds in order that the two wards and the out-patient departmant, which have been of necessity closed, may be reopened. The hospital's building debt is about $£ 5000$ and there is a deficit of half that sum on the current account. 